\title{
Differences in Speed and Power Capacities Between Female National College Team and National Olympic Team Handball Athletes
}

\author{
by \\ Lucas A. Pereira ${ }^{1}$, César C. Cal Abad ${ }^{1}$, Ronaldo Kobal ${ }^{1}$, Katia Kitamura ${ }^{1}$, \\ Rita C. Orsi², Rodrigo Ramirez-Campillo ${ }^{3}$, Irineu Loturco ${ }^{1}$
}

The aim of this study was to compare and examine differences in several neuromuscular assessments between female national Olympic team (Rio-2016) and national college team handball players (2015-Gwangju Summer Universiade). Twenty-eight elite female handball players of the national Brazilian Olympic $(n=12)$ and college $(n=16)$ teams participated in this study. The Olympic and college athletes performed the following speed-power tests assessing mean propulsive power (MPP) in loaded jump squat (JS) and bench press (BP) exercises, unloaded squat and countermovement jumps (SJ and CMJ), sprint performance over 5-, 10-, and 20-m, and change of direction ability in a standard Zig-zag test and a T-Test. The differences between Olympic and college team performances in all variables were analyzed using the magnitude-based inference. The Olympic group presented likely higher performances in the SJ, $C M J$, and MPP JS and very likely higher performances in the MPP BP and T-Test than the college group. The differences in the linear sprint velocity in 5-, 10-, and 20-m tests as well as in the Zig-zag test were all rated as unclear. These findings may have substantial implications for the development of effective strength-power training and testing strategies in elite handball. In addition, coaches and researchers can use these data to create efficient talent identification programs for youth handball players.

Key words: team-sports, physical performance, muscle power, court-sports, COD ability.

\section{Introduction}

Head coaches and fitness specialists face the challenge of developing multiple physical abilities in elite female team sports. Muscle power and speed are key components of performance in many of these disciplines, especially in handball which is witnessing an increase in rapid accelerations, change of direction (COD) tasks, and explosive actions (e.g., jumps) during training and official competitions (Karcher and Buchheit, 2014; Michalsik et al., 2013; Povoas et al., 2012). To respond to this increased physical demand, it becomes necessary to search for more efficient training approaches, which are able to progressively enhance neuromechanical performance in handball players from less to more specialized competitive levels.

A rational way to develop effective strategies to improve performance in top-level sports is to investigate the main determinants of performance in athletes from different levels or age-categories (Carling et al., 2008; Granados et al., 2013; Kobal et al., 2016; Morais et al., 2014). Indeed, Loturco et al. (2014) demonstrated that senior soccer players could perform better in

\footnotetext{
1 - NAR - Nucleus of High Performance in Sport, São Paulo, SP, Brazil.

2 - Brazilian Handball Confederation, Aracaju, SE, Brazil.

3 - Department of Physical Activity Sciences, Research Nucleus in Health, Physical Activity and Sport, University of Los Lagos, Osorno, Chile.
} 
speed and lower limb power tests than their less specialized peers (i.e., under-20). Kobal et al. (2016) reported that national team rugby union players presented higher scores in vertical jump, speed, and COD measurements compared to less qualified professional club athletes. Similarly, previous investigations have shown significant differences in throwing velocity and muscle power (but not in jump and sprint capacities) between male and female handball players from distinct competitive levels (Gorostiaga et al., 2005; Granados et al., 2007, 2013). Nevertheless, these studies did not assess (nor compared) the COD ability of players, which has been recognized as one of the most important factors influencing team sport performance (Iacono et al., 2015; Karcher and Buchheit, 2014; Mclellan et al., 2011; Povoas et al., 2012). Hence, it would be of great interest to perform more comprehensive comparisons between players with different training experiences (e.g., Olympic versus national college team athletes) to determine whether these complex physical abilities are able to distinguish between their competitive skills.

Despite their inherent methodological limitations (e.g., impossibility of inferring causality), the use of comparative cross-sectional studies may have important implications in sport settings, guiding coaches in their daily work and providing scientists with meaningful information for developing further research (Carling, 2017). Therefore, the aim of this study was to compare and examine the differences in a wide range of neuromechanical assessments (i.e., vertical jumps, linear sprint speed, and COD ability) between female national Olympic team (Rio-2016) and national College team handball players (2015Gwangju Summer Universiade). Taking into consideration the previous investigations on this topic and our extensive experience with Olympic athletes, we expected that the Olympic team would demonstrate higher levels of efficiency in all performance measurements.

\section{Methods}

\section{Participants}

Twenty-eight elite female handball players from the national Brazilian Olympic $(\mathrm{n}=$ 12) and college $(n=16)$ teams participated in this study. The characteristics of the subjects are presented in Table 1. The Olympic team was the overall World Champion in Servia-2013 and the college team took $5^{\text {th }}$ place in the 2015 Summer Universiade in Gwangju, South Korea, thus attesting the high level of competitiveness of the players. Before participating in this study, athletes signed an informed consent form. The study was approved by the Anhanguera-Bandeirante University Ethics Committee.

\section{Procedures}

The Olympic and college teams performed the speed-power tests 1-week apart as follows: (1) mean propulsive power (MPP) in loaded jump squat (JS) and bench press (BP) exercises, (2) unloaded squat and countermovement jumps (SJ and CMJ), (3) sprint performance over 5-, 10-, and 20-m, and (4) COD ability in a standard Zig-zag test and a T-Test. All tests were conducted by an experienced evaluator, and the players were previously familiarized with the testing procedures, which were all carried out during a training camp period. During the training camp, training routines, sleep time, and nutrition habits were organized and controlled by the technical staff on the days prior to testing. Before the tests (performed on the same day) the athletes performed $20 \mathrm{~min}$ of a general and specific warm-up, including moderate running (10 $\mathrm{min})$, active stretching $(5 \mathrm{~min})$, and submaximal jumps. The muscle power tests were selected based on their relationship with the actual performance of handball players during training and matches (Karcher and Buchheit, 2014; Povoas et al., 2012) (e.g., association between throwing velocity and power output in benchpress exercise). COD tests were used due to the high frequency and crucial importance of COD during official handball matches (Karcher and Buchheit, 2014).

Vertical Jump Tests

For the SJ, athletes were required to remain in a static position with a $90^{\circ}$ knee flexion angle for $\sim 2 \mathrm{~s}$ before jumping, without any preparatory movement. During the CMJ, athletes were instructed to execute a downward movement to a self-selected height followed by full extension. The SJ and CMJ were executed with the hands on the hips. All jumps were performed on a contact mat (Smart Jump; Fusion Sport, Coopers Plains, Brisbane, Australia) for calculation of jump height based on flight time. A total of five valid attempts (i.e., executed with 
proper jump technique) were allowed for each jump, interspersed by 15-s intervals, and the highest jumps for the $\mathrm{SJ}$ and $\mathrm{CMJ}$ were used for further analysis. The intraclass coefficient of correlation (ICC) values were $\geq 0.96$ for the SJ and $\mathrm{CMJ}$ in both groups of players.

Change of direction tests

The Zig-zag COD test was performed on an indoor court and consisted of four 5-m sections (total 20-m linear distance) marked with cones set at $100^{\circ}$ angles (Figure 1), requiring athletes to decelerate and accelerate as fast as possible around each cone. Two maximal attempts were performed with a 5-min rest interval in-between. Starting from a standing position with the front foot placed 0.3-m behind the first pair of timing gates (Smart Speed, Fusion Equipment, Brisbane, Australia) (i.e., starting line), the athletes were instructed to complete the test as quickly as possible, before crossing the second pair of timing gates, placed 20-m from the starting line (Little and Williams, 2005). The fastest time from the two attempts was retained for further analysis.

The T-Test (Semenick, 1990) was also performed on an indoor court, starting $0.3-\mathrm{m}$ behind a pair of timing gates (Smart Speed, Fusion Equipment, Brisbane, Australia). The athletes began by running forward 9.14-m, then they touched a cone with their hand and moved $4.57-\mathrm{m}$ to the left in lateral shuffling and touched another cone. Next, they moved 9.14-m to the right in lateral shuffling and touched a cone. Finally, the athletes moved to the left by $4.57-\mathrm{m}$, still in lateral shuffling, touched a cone and ran backwards further 9.14-m in the direction of the starting line to finish the test. The test was repeated if an athlete failed to touch a cone or crossed his or her feet during the sidestep phases. The fastest time from the two trials was retained for further analysis.

Sprinting speed and calculation of the COD deficit

For assessment of sprint velocity, four pairs of timing gates (Smart Speed, Fusion Equipment, Brisbane, Australia) were positioned at distances of 0-, 5-, 10- and 20-m on an indoor running track. Each athlete started from a standing position $0.3-\mathrm{m}$ behind the start line. A 5min rest interval was allowed between the two attempts and the fastest time was used for further analysis. To evaluate the efficacy of the individual's ability to utilize their sprint speed during a COD task, a modified COD deficit was calculated based on prior research (Nimphius et al., 2016). The current study presented the COD deficit as a velocity measure calculated as follows: $\{20-m$ velocity - Zig-zag test velocity $\}$. This measure provides information on how much of the individual's maximal sprint velocity could be used or maintained despite the changes of direction required during the (Zig-zag) COD test which was also 20-m in length (Nimphius et al., 2016).

Mean propulsive power in jump squat and bench press exercises

Mean propulsive power in the JS and BP exercises was assessed on a Smith machine (Hammer Strength, Rosemont, IL, USA). Athletes were instructed to execute two repetitions at maximal velocity for each load, starting at $40 \%$ and $30 \%$ of their body mass (BM) for the JS and $\mathrm{BP}$, respectively. For the JS, athletes performed a countermovement until the thigh was parallel to the ground $\left(\sim 100^{\circ}\right.$ relative knee angle) and, after a command, jumped as fast as possible without losing contact between their shoulder and the bar. During the $\mathrm{BP}$, the athletes were instructed to lower the bar in a controlled manner until the barbells lightly touched their chest and then to push the bar as fast as possible. A load of $10 \%$ of $\mathrm{BM}$ for the JS and $5 \%$ of $\mathrm{BM}$ for the $\mathrm{BP}$ was gradually added until a decrease in MPP was observed. A 5-min rest interval between trials was provided. To determine MPP, a linear transducer sampling at $1000 \mathrm{~Hz}$ (T-Force, Dynamic Measurement System; Ergotech Consulting S.L., Murcia, Spain) was attached to the Smith machine bar. The technical specification of the MPP analysis, its calculation, and the use of the MPP rather than peak power have been described previously (Loturco et al., 2015, 2017a, b; SanchezMedina et al., 2010). The maximum MPP value obtained was used for subsequent data analysis. To avoid misinterpretation of the power outputs, these values were normalized by dividing the absolute power value by the body mass (i.e., relative power $=\mathrm{W} \cdot \mathrm{kg}^{-1}$ ). The ICC values observed for MPP in the JS and BP exercises were $\geq 0.92$ in both groups of players.

\section{Statistical Analysis}

Data are presented as means and standard deviations. The normality of data was confirmed using the Shapiro-Wilk test. The differences 
between Olympic and college performances in all variables were analyzed using the magnitudebased inference (Batterham and Hopkins, 2006). The magnitude of the between-group differences in the performance variables was expressed as standardized mean differences (Cohen's $d$ ). The smallest worthwhile change was set using the Cohen's principles for a small effect size (ES: 0.2) for each variable tested (Cohen, 1988). The quantitative chances of finding differences in the variables tested were assessed qualitatively as follows: $<1 \%$, almost certainly not; 1 to $5 \%$, very unlikely; 5 to $25 \%$, unlikely; 25 to $75 \%$, possible; 75 to $95 \%$, likely; 95 to $99 \%$, very likely; $>99 \%$, almost certain. A meaningful difference was considered using the mechanistic inference, based on threshold chances of $5 \%$ for substantial magnitude (Hopkins et al., 2009). Therefore, if the chances of having better and poorer results were both $>5 \%$, the true difference was assessed as unclear. Additionally, the magnitude of the standardized differences was interpreted using the following thresholds: $<0.2,0.2-0.6,0.6-1.2,1.2-$ 2.0, 2.0-4.0, and $>4.0$ as trivial, small, moderate, large, very large, and near perfect, respectively (Hopkins et al., 2009). The ICC values were used to indicate the relationship within vertical jumps and muscle power tests, for height and MPP. Finally, the coefficient of variation $(\mathrm{CV} \%)$ and the typical error of measurement (TE) were calculated in all variables tested and for both groups of players, following the principles established by Hopkins (2004).

\section{Results}

The Olympic athletes were older and taller than their college peers, although no significant differences were observed for BM (Table 1). The $\mathrm{CV}$ and $\mathrm{TE}$ for each variable assessed in the Olympic and college groups are presented in Table 2.

Figure 2 depicts the standardized differences in the muscle power and vertical jump assessments between national Olympic and college handball teams. The Olympic group presented likely higher performances in the SJ $(32.3 \pm 4.4 \mathrm{~cm}$ vs. $30.0 \pm 5.6 \mathrm{~cm}$, Cohen's $d[90 \%$ confidence limits]: 0.54 [-0.07; 1.15]), CMJ (32.9 \pm $3.9 \mathrm{~cm}$ vs. $30.9 \pm 5.8 \mathrm{~cm}, 0.52[-0.11 ; 1 ; 14])$, and MPP JS $\left(7.84 \pm 1.56 \mathrm{~W} \cdot \mathrm{kg}^{-1}\right.$ vs. $6.99 \pm 0.98 \mathrm{~W} \cdot \mathrm{kg}^{-1}$, $0.66[-0.15 ; 1.47])$, and very likely higher in the MPP BP $\left(5.15 \pm 0.82 \mathrm{~W} \cdot \mathrm{kg}^{-1}\right.$ vs. $4.39 \pm 0.70 \mathrm{~W} \cdot \mathrm{kg}^{-1}$, $1.02[0.39 ; 1.65])$ compared to the college group.

Table 1

Characteristics of the handball players for the Olympic and college groups.

Olympic College $\quad \begin{gathered}\text { Percent chances of finding } \\ \text { differences between groups }\end{gathered} \quad \begin{gathered}\text { Standardized mean } \\ \text { differences (90\% CL) }\end{gathered}$

\begin{tabular}{lcccc}
\hline Age (years) & $27.6 \pm 4.6$ & $22.9 \pm 2.2$ & $100 / 00 / 00$ Almost certain & $1.33(0.69 ; 1.97)$ Large \\
Body height $(\mathrm{cm})$ & $176.3 \pm 5.7$ & $170.9 \pm 5.0$ & $99 / 01 / 00$ Very likely & $1.26(0.49 ; 2.03)$ Large \\
Body mass $(\mathrm{kg})$ & $68.3 \pm 7.5$ & $69.2 \pm 8.4$ & $18 / 38 / 44$ Unclear & $0.15(-0.48 ; 0.77)$ Trivial \\
\hline
\end{tabular}

Data are presented as mean \pm standard deviation; CL: confidence limits. 
Table 2

Coefficient of variation (CV) and typical error of measurement (TE) in the test variables for the Olympic and college groups.

\begin{tabular}{lcccc}
\hline & \multicolumn{2}{c}{ Olympic } & \multicolumn{2}{c}{ College } \\
\cline { 2 - 5 } & CV\% & TE & CV\% & TE \\
\hline SJ & 3.1 & 0.69 & 3.6 & 0.72 \\
CMJ & 2.8 & 0.61 & 3.3 & 0.67 \\
MPP JS & 7.1 & 0.37 & 6.3 & 0.29 \\
MPP BP & 6.8 & 0.19 & 5.4 & 0.15 \\
VEL 5-m & 1.8 & 0.01 & 2.4 & 0.02 \\
VEL 10-m & 1.3 & 0.02 & 1.6 & 0.02 \\
VEL 20-m & 1.0 & 0.02 & 1.1 & 0.03 \\
Zig-zag & 1.0 & 0.04 & 1.3 & 0.05 \\
T-Test & 1.5 & 0.11 & 1.8 & 0.14 \\
\hline
\end{tabular}

SJ: squat jump; CMJ: countermovement jump; MPP: mean propulsive power; JS: jump squat; BP: bench press; VEL: sprint velocity.

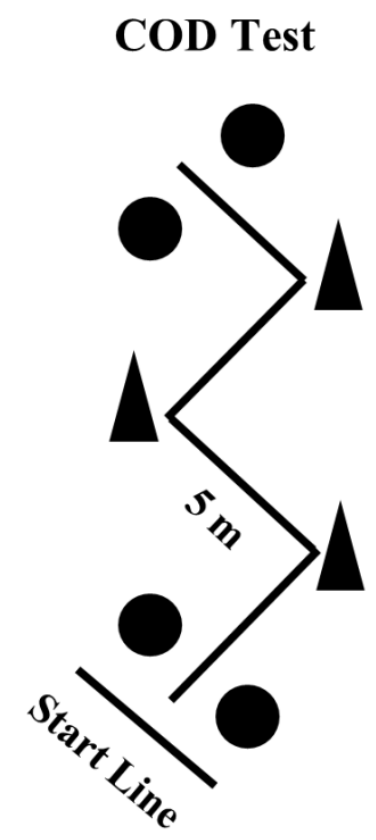

Figure 1

A schematic presentation of the Zig-zag test. Circles represent the position of the timing gates. 

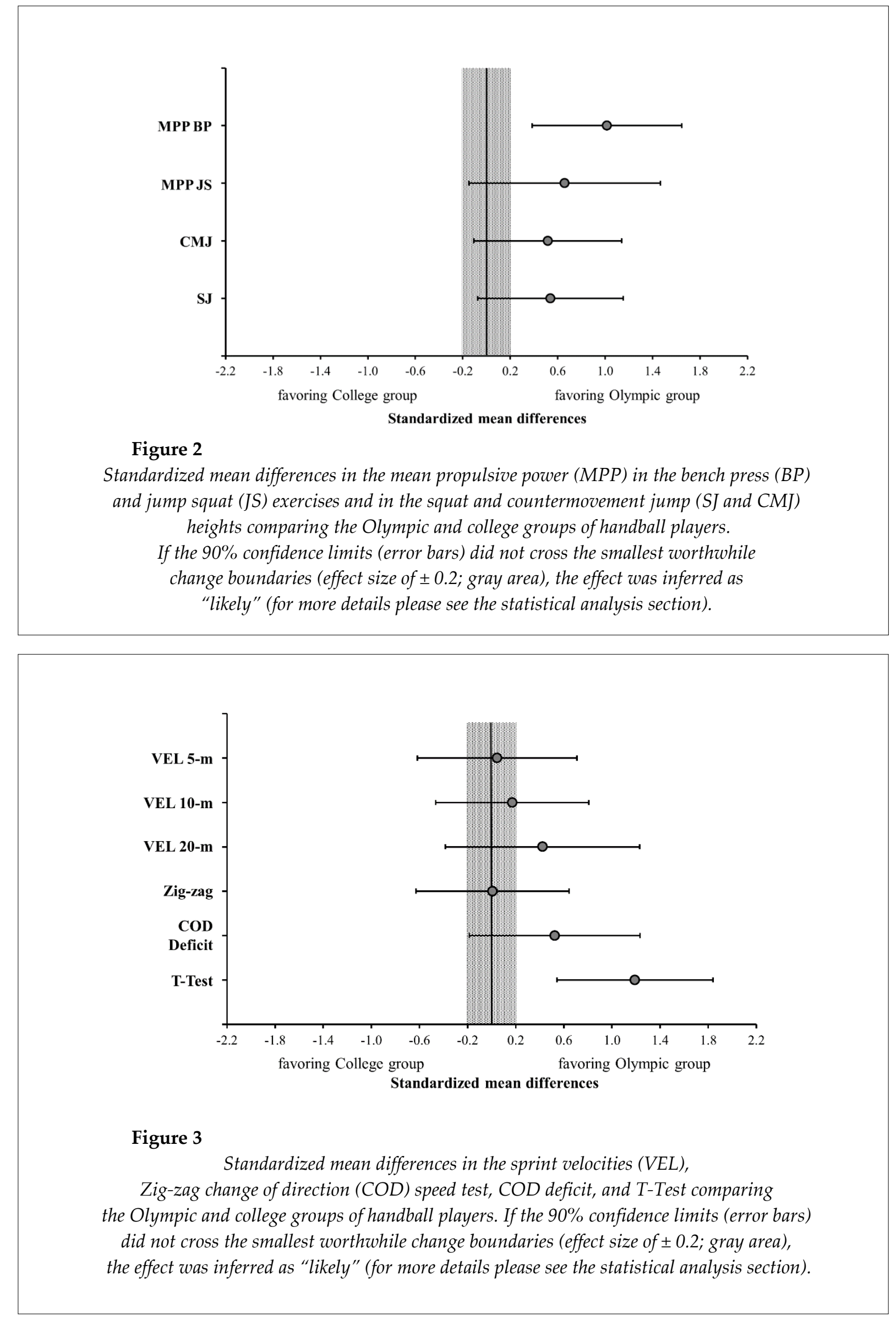
Figure 3 demonstrates the standardized differences in the linear sprint, Zig-zag test, COD deficit, and T-Test results between Olympic and college groups. The college group presented a likely lower COD deficit than the Olympic group $\left(2.64 \pm 0.19 \mathrm{~m} \cdot \mathrm{s}^{-1}\right.$ vs. $2.73 \pm 0.16 \mathrm{~m}^{-1}, 0.52$ [-0.19; 1.23]). The Olympic group showed a very likely higher performance in the T-Test than the college group $\left(3.55 \pm 0.19 \mathrm{~m} \cdot \mathrm{s}^{-1}\right.$ vs. $3.36 \pm 0.15 \mathrm{~m} \cdot \mathrm{s}^{-1}, 1.19$ [0.54; 1.84]). The differences in the linear sprint velocity in 5-, 10-, and 20-m tests and in the Zigzag test were all rated as unclear (VEL 5-m: $4.69 \pm$ $0.26 \mathrm{mss}^{-1}$ vs. $4.68 \pm 0.26 \mathrm{~m} \cdot \mathrm{s}^{-1}, 0.05$ [-0.62; 0.71$]$;

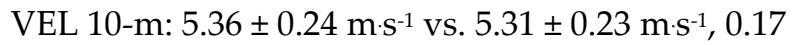
[-0.47; 0.81]; VEL 20-m: $6.16 \pm 0.25 \mathrm{~m} \cdot \mathrm{s}^{-1}$ vs. $6.08 \pm$

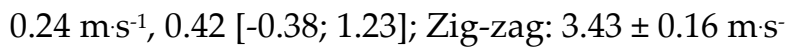
${ }^{1}$ vs. $3.44 \pm 0.10 \mathrm{~ms}^{-1}, 0.01$ [-0.63; 0.64]; for Olympic and college groups, respectively).

\section{Discussion}

This study aimed to compare and determine the differences in neuromuscular performance between female national Olympic team and college team handball players. The main findings reported here are that the Olympic group performed better than collegiate players in muscle power assessments (in upper and lower limbs), vertical jump measurements (SJ and CMJ), and the agility T-Test. Unexpectedly, no meaningful differences were observed in the linear sprint velocity across all examined distances (5-, 10-, and 20-m) or Zig-zag test performance.

As expected, Olympic athletes demonstrated greater performance in vertical jump tests and MPP in the JS and BP exercises than the "less qualified" college group. These results are in line with a previous study that found superior vertical jump height and power outputs in the half-squat (HS) and BP exercises when comparing female handball players from distinct competitive levels and training backgrounds (Granados et al., 2013). The same holds true for another study with the same characteristics, which demonstrated differences in power outputs for the same exercises (HS and BP) when comparing amateur and elite female handball players (Granados et al., 2007). It is reasonable to consider that these differences in the ability to produce muscle power may be related to greater levels of performance in some specific game-tasks, such as jumps and ball throws. Accordingly, it has been shown that both lower and upper limb power outputs were positively correlated with the actual throwing velocity of handball players (Granados et al., 2007, 2013). Furthermore, athletes able to jump higher can present an advantage over their adversaries (for example) during offensive game actions, where handball players should jump as high and fast as possible in order to find better opportunities to score a goal (Karcher and Buchheit, 2014). These data may have significant implications for the development of resistance training programs intended for professional handball players and can also be used for improving the accuracy and effectiveness of handball talent identification programs.

Despite the differences in muscle power, the linear sprint velocity was not different between the two groups of players across all the assessed distances. It is worth highlighting that, due to the confined spaces of handball courts (which prevents large player displacements) and the technical-tactical characteristics of the game (e.g., based mostly on attacking vs. defense situations that occur in an area of less than half the court), linear sprints represent a very low percentage of the actions performed by athletes during matches (and specific training sessions) (Karcher and Buchheit, 2014; Povoas et al., 2012). Indeed, it has been shown that during official games elite handball players execute a substantial number of changes of directions, sideway movements, and backward running actions, while performing very limited sets of linear sprints (Michalsik et al., 2013; Povoas et al., 2012). In part, these facts may explain the absence of differences in the linear sprint ability presented by female handball players of different competitive levels, which has also been reported in other studies covering similar populations (Baker and Newton, 2008; Gorostiaga et al., 2005; Granados et al., 2013). Probably, the selection of players more prone to compete at the highest levels of human performance (i.e., Olympic team athletes) relies more on physical traits directly associated with the majority of tasks performed throughout the games (e.g., COD tasks).

In relation to the COD assessments, greater performance was observed for the T-Test when comparing the distinct groups of players, whereas the differences in the Zig-zag test were rated as "unclear". The relative differences 
between the two COD measurements can be explained by the mechanical and technical differences between the drills. While in the T-Test subjects perform four sequential $90^{\circ}$ lateral directional changes, during the Zig-zag test, players must execute three $45^{\circ}$ cutting maneuvers (Figure 1). Due to its complex requirements, it is possible that the T-Test better simulates the actual movement patterns usually performed by handball players (i.e., sideway movements and backward running) (Michalsik et al., 2013). Conversely, the Zig-zag test seems to be more dependent on individual ability to accelerate and decelerate fast, with less accentuated turning angles, and a lower number of turns performed throughout the test (when compared to the TTest). It seems that the T-Test is more suitable to distinguish between players at different competitive levels and from different training backgrounds, and thus should be considered as a potential tool to evaluate and discriminate between top-level handball athletes.

Curiously, the higher performances in vertical jumps, muscle power tests, and the T-Test in the Olympic group were also accompanied by a greater and unexpected deficit in COD performance (i.e., COD deficit). Briefly, the COD deficit was previously defined as "a more isolated measure of COD ability" (Nimphius et al., 2016), since this measure suppresses the effect of linear velocity during the numerical analysis of COD actions. Since COD tasks rely on a wide range of neuromechanical capabilities (Brughelli et al., 2008; Young and Farrow, 2006), it is plausible to assume that the more powerful (and theoretically more technical) Olympic athletes would also present a superior technical-physical capacity to execute COD drills and, therefore, a lower COD deficit than their hypothetically weaker peers (e.g., college team players). Nonetheless, it seems that the Olympic handball players were not capable of efficiently transferring their superior neuromuscular abilities to more complex motor activities (Young and Farrow, 2006). Among other things, this deficit may occur because the "traditional training strategies" (e.g., muscle power training and plyometric exercises) designed to improve strength, power and speed abilities are not equally effective to enhance COD performance. Possibly, the implementation of different training practices (i.e., specific drills with multiple COD tasks, and with programmed and non-programmed change-of-directions) would be useful and more appropriate to increase the level of transference of the basic neuromuscular qualities (e.g., strength, power, and speed) to COD performance. This is especially important in high performance sport settings, due to the wellrecognized importance of COD ability in specific handball performance (Karcher and Buchheit, 2014; Michalsik et al., 2013; Povoas et al., 2012). Further studies should be carried out to assess the efficacy of different mixed training approaches in enhancing agility performance, and thus, reducing the COD deficit.

\section{Practical Implications}

The data reported here indicate that female national Olympic handball players perform better than national collegiate players in a series of muscle power tests and speed-related assessments. Nevertheless, this apparent "physical superiority" is not capable of producing athletes who are more efficient in the rapid execution of changes of directions, which can be confirmed by the greater COD deficit presented by the Olympic group. Therefore, coaches and researchers are encouraged to search for different training schemes and monitoring tools, able to (at the same time) gradually develop speed-power qualities and ensure an effective transference of these abilities to agility performance. These training responses can be constantly assessed using the practical physical tests implemented in this study, allowing frequent and precise adjustments throughout the training process. This information has a significant implication in applied settings, due to the recognized importance of COD speed in handball. The finding that Olympic players can produce more power (in lower and upper limbs) and jump higher than their weaker counterparts should underline the practical relevance of these capabilities in professional handball, which has previously been suggested by other authors (Karcher and Buchheit, 2014; Manchado et al., 2013; Van Den Tillaar, 2004). Finally, as Olympic athletes are considered as individuals at "the extreme edge of human performance", the results demonstrated here may provide valuable information and reference values for players in different stages of development, as well as for specialized talent identification programs. 


\section{References}

Baker DG, Newton RU. Comparison of lower body strength, power, acceleration, speed, agility, and sprint momentum to describe and compare playing rank among professional rugby league players. J Strength Cond Res, 2008; 22: 153-158

Batterham AM, Hopkins WG. Making meaningful inferences about magnitudes. Int J Sports Physiol Perform, 2006; $1: 50-57$

Brughelli M, Cronin J, Levin G, Chaouachi A. Understanding change of direction ability in sport: a review of resistance training studies. Sports Med, 2008; 38: 1045-1063

Carling C. Scientific research in association football - editors and reviewers get of my case (study). Sport Perform Sci Reports, 2017; 1: 1-3

Carling C, Bloomfield J, Nelsen L, Reilly T. The role of motion analysis in elite soccer: contemporary performance measurement techniques and work rate data. Sports Med, 2008; 38: 839-862

Cohen J. Statistical power analysis for the behavioral sciences. Hillsdale (NJ): Lawrence Erlbaum Associates; 1988

Gorostiaga EM, Granados C, Ibanez J, Izquierdo M. Differences in physical fitness and throwing velocity among elite and amateur male handball players. Int J Sports Med, 2005; 26: 225-232

Granados C, Izquierdo M, Ibanez J, Bonnabau H, Gorostiaga EM. Differences in physical fitness and throwing velocity among elite and amateur female handball players. Int J Sports Med, 2007; 28: 860-867

Granados C, Izquierdo M, Ibanez J, Ruesta M, Gorostiaga EM. Are there any differences in physical fitness and throwing velocity between national and international elite female handball players? J Strength Cond Res, 2013; 27: 723-732

Hopkins WG. How to interpret changes in an athletic performance test. Sportsci, 2004; 8: 1-7

Hopkins WG, Marshall SW, Batterham AM, Hanin J. Progressive statistics for studies in sports medicine and exercise science. Med Sci Sports Exerc, 2009; 41: 3-13

Iacono AD, Eliakim A, Meckel Y. Improving fitness of elite handball players: small-sided games vs. highintensity intermittent training. J Strength Cond Res, 2015; 29: 835-843

Karcher C, Buchheit M. On-court demands of elite handball, with special reference to playing positions. Sports Med, 2014; 44: 797-814

Kobal R, Nakamura FY, Moraes JE, Coelho M, Kitamura K, Cal Abad CC, Pereira LA, Loturco I. Physical Performance of Brazilian Rugby Players From Different Age Categories and Competitive Levels. J Strength Cond Res, 2016; 30: 2433-2439

Little T, Williams AG. Specificity of acceleration, maximum speed, and agility in professional soccer players. J Strength Cond Res, 2005; 19: 76-78

Loturco I, Kobal R, Gil S, Pivetti B, Kitamura K, Pereira LA, Abad CC, Nakamura FY. Differences in loaded and unloaded vertical jumping ability and sprinting performance between Brazilian elite under-20 and senior soccer players. Am J Sports Sci, 2014; 2: 8-13

Loturco I, Kobal R, Kitamura K, Fernandes V, Moura N, Siqueira F, Cal Abad CC, Pereira LA. Predictive factors of elite sprint performance: influences of muscle mechanical properties and functional parameters. J Strength Cond Res, 2017a; In Press:

Loturco I, Nakamura FY, Tricoli V, Kobal R, Abad CC, Kitamura K, Ugrinowitsch C, Gil S, Pereira LA, Gonzales-Badillo JJ. Determining the optimum power load in jump squats using the mean propulsive velocity. PLoS One, 2015; 10: e0140102

Loturco I, Pereira LA, Moraes JE, Kitamura K, Cal Abad CC, Kobal R, Nakamura FY. Jump-Squat and HalfSquat Exercises: Selective Influences on Speed-Power Performance of Elite Rugby Sevens Players. PLoS One, 2017b; 12: e0170627

Manchado C, Tortosa-Martinez J, Vila H, Ferragut C, Platen P. Performance factors in women's team handball: physical and physiological aspects--a review. J Strength Cond Res, 2013; 27: 1708-1719

Mclellan CP, Lovell DI, Gass GC. Performance analysis of elite rugby league match play using global positioning systems. J Strength Cond Res, 2011; 25: 1703-1710

Michalsik LB, Aagaard P, Madsen K. Locomotion characteristics and match-induced impairments in physical performance in male elite team handball players. Int J Sports Med, 2013; 34: 590-599 
Morais JE, Marques MC, Marinho DA, Silva AJ, Barbosa TM. Longitudinal modeling in sports: young swimmers' performance and biomechanics profile. Hum Mov Sci, 2014; 37: 111-122

Nimphius S, Callaghan SJ, Spiteri T, Lockie RG. Change of Direction Deficit: A More Isolated Measure of Change of Direction Performance Than Total 505 Time. J Strength Cond Res, 2016; 30: 3024-3032

Povoas SC, Seabra AF, Ascensao AA, Magalhaes J, Soares JM, Rebelo AN. Physical and physiological demands of elite team handball. J Strength Cond Res, 2012; 26: 3365-3375

Sanchez-Medina L, Perez CE, Gonzalez-Badillo JJ. Importance of the propulsive phase in strength assessment. Int J Sports Med, 2010; 31: 123-129

Semenick D. Tests and measurements: The T-Test. Strength Cond J, 1990; 12: 36-37

van den Tillaar R. Effect of different training programs on the velocity of overarm throwing: a brief review. $J$ Strength Cond Res, 2004; 18: 388-396

Young W, Farrow D. A review of agility: practical applications for strength and conditioning. Strength Cond J, 2006; 28: 24-29

\section{Corresponding author:}

\section{Irineu Loturco}

NAR - Nucleus of High Performance in Sport.

Av. Padre José Maria, 555 - Santo Amaro,

04753-060 - São Paulo, SP, Brazil.

Tel.: +55-11-3758-0918

E-mail: irineu.loturco@terra.com.br 